\title{
Development of Whisker Inspired 3D Multi-Material Printed Flexible Tactile Sensors
}

\author{
Bram Eijking, Remco Sanders and Gijs Krijnen \\ Robotics And Mechatronics group \\ University of Twente \\ Enschede, The Netherlands \\ Email: gijs.krijnen@utwente.nl
}

\begin{abstract}
In this paper a bio-inspired tactile whisker sensor made by 3D printing is discussed. Whisker inspired sensors were printed using flexible dielectric and conductive thermoplastic poly-urethane (TPU). The conductive TPU is used for straingauges facilitating resistive read-out. A single whisker sensor contains two strain-gauges allowing to determine both the force acting on the whisker as well as the contact point on the whisker. To the best of our knowledge this is the first work on 3D multimaterial printed, flexible, whisker inspired tactile sensors.
\end{abstract}

Keywords - Tactile sensor; Elastomer; TPU; 3d multimaterial printing; Fused Deposition Modeling

\section{INTRODUCTION}

Animals like seals and rodents make use of whiskers for sensing their surroundings. These tactile senses have been studied in detail and it is shown that this principle can be used in active sensing [1]. Whiskers can be used for sensing in surroundings were other sensor techniques are not applicable such as in darkness and environments were noise has a big influence. Previous publications have shown the capabilities of using whiskers for applications such as contact imaging [2] and three dimensional position measurements [3]. Previous publications have also explored the possibilities of 3D printing whisker sensors [4], for this whisker one material was used and read-out was done using PCB-based capacitive sensing.

In this paper the first steps towards designing and 3D multimaterial printing a whisker sensor using resistive sensing are shown. The design is aimed at measuring the force applied to the whisker as well as the position at which it acts. The first designs are focussed on 1D measurements with the possibility of increasing to $2 \mathrm{D}$, which would require two additional measurements of force and moment. The reason that $3 \mathrm{D}$ printed sensors are desirable is that the sensors can be integrated directly into the printed design, whereas other fabrication processes require multiple steps to accomplish the same result.

\section{DESIGN}

The function of the whisker inspired tactile sensor is to determine both the force $F_{\text {ext }}$ acting on the whisker as well as its point of action $s$. Using the lever of the whisker the moment $\vec{s} \times \vec{F}_{\text {ext }}$ can be measured from the rotation of the whisker in its elastic socket. The horizontal component of the force $F_{\mathrm{x}}$ can be measured by the displacement along the $x$ direction due to the compliance of the socket in this direction, see Fig. 1.

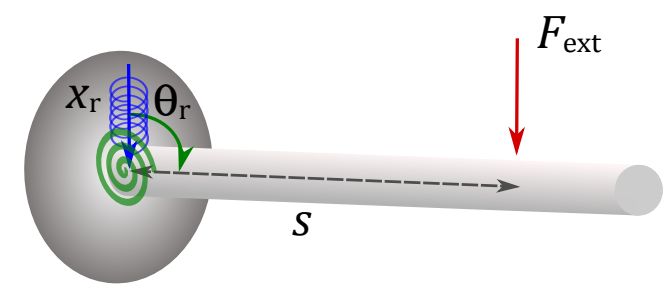

Fig. 1. Schematic of the proposed whisker inspired tactile sensor with rotational stiffness (green) and translational stiffness (blue).

Given the values of the conductivity it is reasonable to use the conductive TPU as strain-gauge in the design. Since the sensor has to enable the determination of both the rotation and displacement of the whisker two strain-gauges are needed to determine both degrees of freedom (DOF).

\section{A. Initial Designs}

Some design ideas were proposed and an initial design was made. The design includes a whisker which is printed in the vertical (i.e. the $z$ ) direction. The strain gauges are printed in the base of the whisker, which is supported to ensure only rotation of the cross-bar and prevent deflection of the endpoints of the strain gauges. A picture of this design is shown in Fig. 2

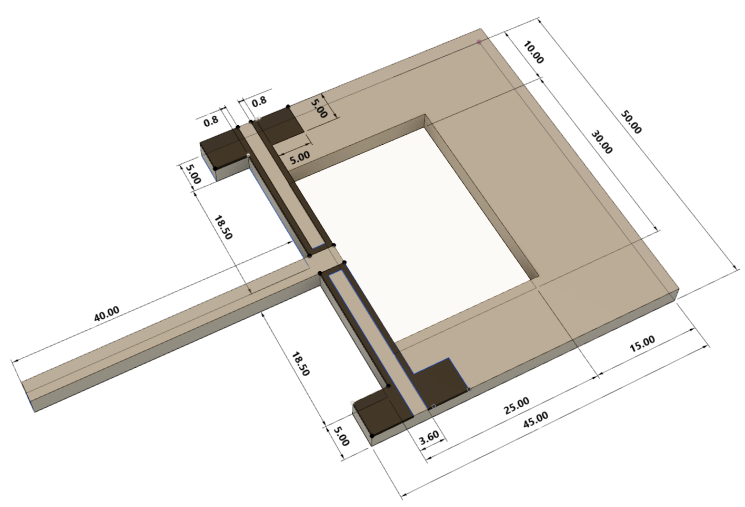

Fig. 2. Sensor design with strain gauges in black. Measures in mm's. 
Some initial measurements on the resistance of the conductive material showed that the magnitude of the resistance is not strongly dependent on the thickness of the layer. This implies that the design of the strain gauges could be based on thin layers. Furthermore it was observed that the resistance in the $z$-direction of the material is much larger than along the $x$ or $y$-directions. Therefore the strain gauges were implemented in structures of only two layers thick, printed in the $x-y$ plane.

\section{MATERIALS USED}

\section{A. Flexible Materials}

For the whisker two flexible materials are used. The first is Ninjaflex [5], this is a flexible non-conductive material and is used for the whisker and base of the design. The second material is a conductive TPU, PI-ETPU 95-250 Carbon Black [6]. It is a flexible material with low conductivity $(<300 \Omega \mathrm{cm})$ based on carbon black fillers and can be $3 \mathrm{D}$ printed.

\section{B. $3 D$ Printing}

The sensors are printed on a Flashforge Creater Pro printer [7] which was adapted specifically for printing of highly flexible filaments by equipping it with a Flexion extruder from Diabase Engineering [8]. The Ninjaflex material was printed with a layer thickness of $200 \mu \mathrm{m}$, a nozzle of $0.6 \mathrm{~mm}$ at a temperature of $220^{\circ} \mathrm{C}$, a hot-bed temperature of $50^{\circ} \mathrm{C}$ and a printing speed of $33.3 \mathrm{~mm} \mathrm{~s}^{-1}\left(2000 \mathrm{~mm} \mathrm{~min}^{-1}\right)$. For the PI-ETPU a layer thickness of $200 \mu \mathrm{m}$ and a nozzle of $0.8 \mathrm{~mm}$ at a temperature of $230^{\circ} \mathrm{C}$ was used. A picture of the printed whisker is shown in figure 3 .

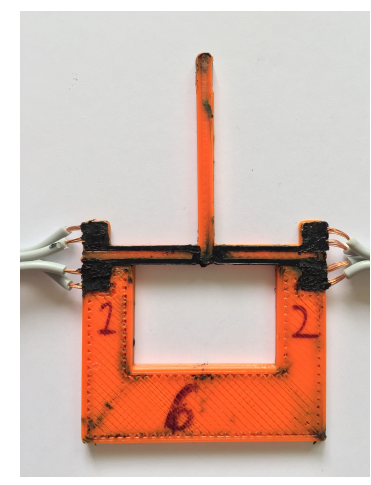

Fig. 3. Printed whisker.

\section{ELECTRICAL CHARACTERIZATION}

The first property analysed is the resistance of the PI-ETPU. This is the most relevant property of the material since it is used for the strain-gauges. Several samples were measured by 4-probe method. Variations in thickness at fixed length $(40 \mathrm{~mm})$ and width $(11 \mathrm{~mm})$ were printed to measure the resistance for given thickness and to compare the results to the resistivity of the material as given by the manufacturer. This showed that under the applied printing conditions, contrary to expectations, there was no clear dependence of the resistance on the thickness of the samples, see Fig. 4.

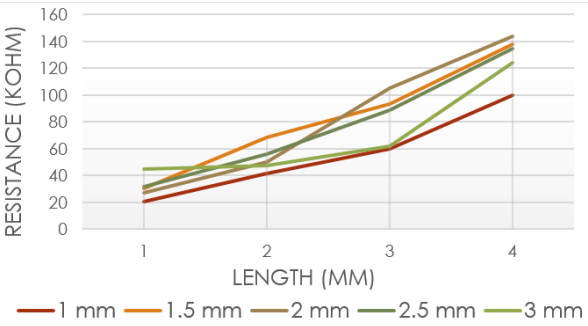

Fig. 4. Resistance vs. length for different thicknesses.

Therefore measurements were done using samples that have only several layers of conductive material. The layer thickness was kept constant at $0.2 \mathrm{~mm}$. For this set of measurements a new setup was used allowing to measure the supplied current and resistance concurrently. The current was stepped from $-100 \mu \mathrm{A}$ to $100 \mu \mathrm{A}$ to see if there was a current dependence of the resistance, Fig. 5. Resistances determined from the slope of the voltage-current relations helped to exclude non-linear or contact effects. The fact that there was no clear relation

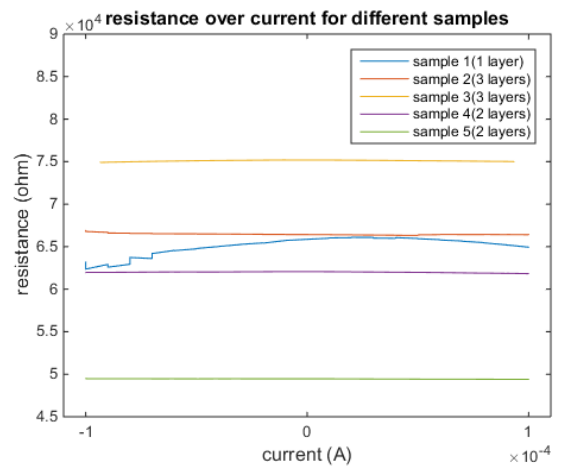

Fig. 5. Resistance measurements with an increasing number of layers.

between resistance and resistor cross-section suggested a large influence of surface conduction. Therefore an experiment was done where the resistance was measured over a period of a few hours after the sample was placed in a nitrogen environment. These measurements showed no clear variation with time making surface conduction less probable.

\section{MECHANICAL CHARACTERIZATION}

\section{A. Model}

In order to make a mechanical model describing the forces and moments acting on the whisker some assumptions are made. 1) The mechanical response can be modelled as a combination of two separate parts, one describing the whisker itself and one describing the base of the whisker. 2) The whisker can be modelled as a cantilever with fixed connection to the base of the whisker. 3) The base of the whisker can be modelled as a clamped-clamped beam, loaded by the combination of a moment and a lateral force in the midpoint of the beam. The remaining forces and moments acting on the beam are the reaction forces and moments. From the model of 
the base of the sensor the displacement under a certain applied force at a certain distance along the whisker is determined.

$$
\begin{aligned}
w(x) & =\frac{M}{E I}\left(\frac{1}{4 L} x^{3}-\frac{1}{8} x^{2}\right) & & \left(x \leq \frac{L}{2}\right) \\
& =\frac{M}{E I}\left(-\frac{1}{4 L}(L-x)^{3}+\frac{1}{8}(L-x)^{2}\right) & & \left(x \geq \frac{L}{2}\right)
\end{aligned}
$$

where $I$ is the 2 nd moment of area of the beam, and $M=$ $S \cdot F \cdot \sin (\theta)$ is the applied moment. This curvature is related to the strain in each of the two strain gauges from which the resistance change is calculated using the gauge-factor (GF). Eventually this analysis results in expressions:

$$
\begin{aligned}
& \frac{\Delta R_{\mathrm{k}}}{R_{\mathrm{k}}}=G F \cdot 2.86 \times 10^{-3}\left(\frac{L}{E I}\right)^{2} M^{2} \pm \frac{G F}{A E} F_{\mathrm{ext}} \\
& \text { with } k=\{\mathrm{L}, \mathrm{R}\}
\end{aligned}
$$

where L, R are used to indicate the left and right strain-gauges respectively. With this expression the moment and force can be derived from

$$
M=\sqrt{\frac{\frac{\Delta R_{\mathrm{L}}}{R_{\mathrm{L}}}+\frac{\Delta R_{\mathrm{R}}}{R_{\mathrm{R}}}}{2 C_{\mathrm{M}}}} \quad F_{\mathrm{ext}}=\frac{\frac{\Delta R_{\mathrm{L}}}{R_{\mathrm{L}}}-\frac{\Delta R_{\mathrm{R}}}{R_{\mathrm{R}}}}{2 C_{\mathrm{F}}}
$$

through measurement of the output of the strain-gauges. In order to complete the model gauge-factors need to be determined. Then using equation 3 the force and moments are calculated from the measured resistance, the distance at which the force acts can then be derived from the relation between the force and moment.

\section{B. Measurements}

Experiments were conducted to validate the mechanical model. In these experiments a linear actuator (SMAC LCA25050-15F, [9]) is used to impose a loading on the whisker while a load-cell is used to simultaneously measure the force. In the first measurements position control was used on the actuator to step the whisker displacement at the contact point from $0 \mathrm{~cm}$ to $2.5 \mathrm{~cm}$ in both directions. The resistance is measured using a 4-point measurement and logged. The measured resistance is plotted against time and the applied force on the whisker, see Fig. 6. The measurements show strong drift (upper left),
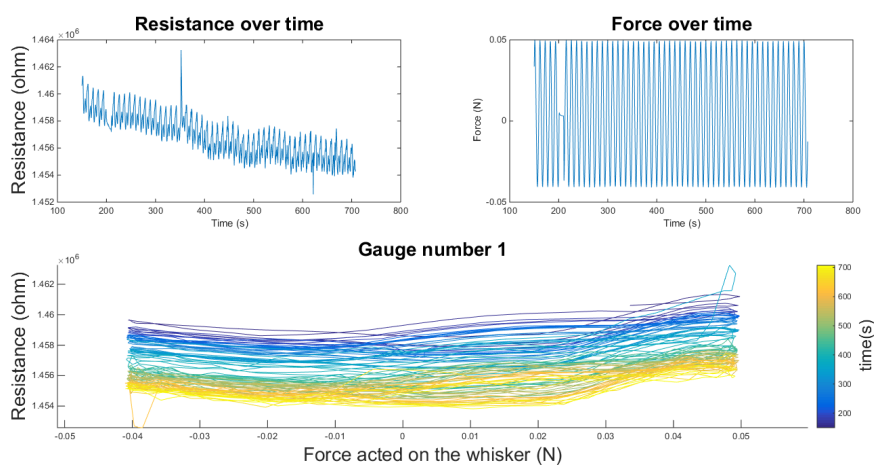

Fig. 6. Resistance measurements on the second design.
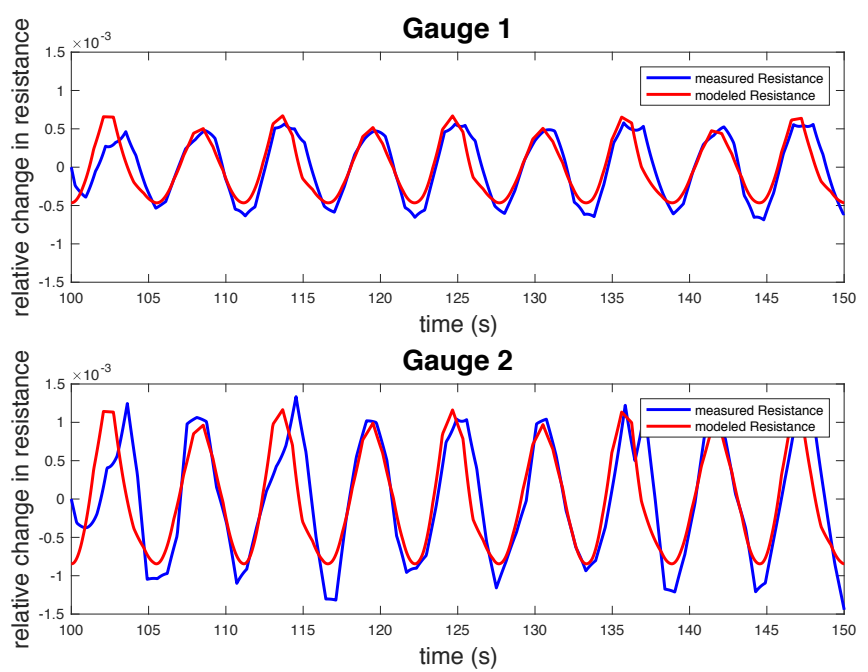

Fig. 7. Measured and modelled resistance versus time after high-pass filtering.

which is seen to level off somewhat at longer times. However, a considerable hysteresis remains observable at all times (bottom). Also the overall response shows asymmetric and nonlinear behaviour, which may be expected from (2).

On a second set of measurements we applied a highpass filter to reduce the drift component. A second degree polynomial fit was used to determine the parameters needed for the model. The result was plotted and compared to the measurements, Fig. 7. The model reasonably predicts the difference between positive and negative forces as well as the differences between both gauges.

\section{CONCLUSIONS}

We have proposed a 3D multi-material printed whisker inspired flexible tactile sensors made from a combination of dielectric and conductive TPU. Initial designs and measurements were presented. A linearised model was introduced to gain a first quantitative understanding of the devices.

\section{REFERENCES}

[1] Hartmann, M.J., Active Sensing Capabilities of the Rat Whisker System, Autonomous Robots (2001) 11: 249. doi:10.1023/A:1012439023425

[2] Tyler N. Clements and Christopher D. Rahn, Three-Dimensional Contact Imaging with an Actuated Whisker, IEEE Transactions on Robotics, Vol22, 2006, DOI: 10.1109/TRO.2006.878950.

[3] Ozkan Bebek, M. Cenk Cavusoglu, Whisker Sensor Design for Three Dimensional Position Measurement in Robotic Assisted Beating Heart Surgery, Proc. IEEE Int. Conf. Robotics and Automation, 2007, DOI: 10.1109/ROBOT.2007.363791.

[4] J. Delamare, R. Sanders and G. Krijnen, "3D printed biomimetic whisker-based sensor with co-planar capacitive sensing," 2016 IEEE SENSORS, Orlando, FL, 2016, pp. 1-3, DOI: 10.1109/ICSENS.2016.7808631.

[5] Palmiga Innovations, Material info for PI-ETPU 95-250 Carbon Black, Online: http://rubber3dprinting.com/pi-etpu-95-250-carbon-black/ last visited June 2017.

[6] Ninjatek, NinjaFlex 3D Printing Filament Technical Specifications, https://ninjatek.com/wp-content/uploads/2016/05/NinjaFlex-TDS.pdf

[7] Online: http://www.flashforge.com/creator-pro-3d-printer/ last visited June 2017.

[8] Online: https://flexionextruder.com last visited June 2017.

[9] Online: http://www.smac-mca.com/index.php last visited June 2017. 